\title{
Spread-of-Disease Modeling in a Microbiology Course
}

\author{
George W. Shiflet and Angela B. Shiflet \\ Wofford College, Spartanburg, South Carolina, USA \\ \{shifletgw, shifletab\}@wofford. edu \\ http: / / www. wofford. edu/ecs /
}

\begin{abstract}
Microbiology is the study of microorganisms. Most college courses in microbiology emphasize the biology of bacteria and viruses, including those that are human pathogens. One challenging aspect of the course is to introduce students to epidemiology, which considers the causes, dispersal, and control of disease. Although disease transmission models have helped develop successful strategies for managing epidemics, most science students are unaware of their advantages and complexities. To address this challenge, the microbiology course at Wofford College has incorporated a sequence of three or four laboratories on modeling the spread of disease. Emphasis in Computational Science students who have studied modeling and simulation in depth serve as laboratory assistants and mentors. Evidence from test scores and self-assessment support the hypothesis that the sequence of laboratories has improved student understanding of human disease dynamics and demonstrated the utility of computational models.
\end{abstract}

Keywords: computational science, education, modeling, microbiology, spread of disease.

\section{Introduction}

Many institutions of higher education offer a junior/senior level microbiology course to study of the biology bacteria, fungi and viruses. Although these microorganisms are important part of various ecosystems, many can cause devastating, infectious diseases. Thus, in a course that emphasizes human disease, having epidemiology as a component of the course is essential. Epidemiology is the study of the causes, dispersal, and control of disease.

Computational models of disease transmission have been instrumental in designing successful strategies for managing epidemics for a number of diseases. For example, Marc Lipsitch in collaboration with others developed a model for the spread of Severe Acute Respiratory Syndrome (SARS) and used the model to make predictions on the impact of public health efforts to reduce disease transmission [1]. As another example, using data and mathematical models, the Dutch Ministry of Health, Welfare and Sports developed "a national plan to minimize effects of pandemic influenza" [2]. Recognizing the benefits of modeling the spread of disease, the National Institute of General Medical Sciences, one of the National Institutes of Health, has a collaborative effort, Models of Infectious Disease Agent Study (MIDAS), to develop computational 
models for use by "policymakers, public health workers, and other researchers who want to better understand and respond to emerging infectious diseases" [3].

To help students appreciate some of the techniques, challenges, and benefits of computational spread-of-disease models, for three years biologist George Shiflet has incorporated a sequence of laboratories into Microbiology, a class with 30 to 40 students, at Wofford College [4]. After a tutorial on using a systems dynamics software tool, students in pairs investigate various diseases, develop models of the spread of those diseases, present their work, and write an analysis of the results.

\section{Tutorial in Laboratory 1}

System dynamics models provide global views of major systems that change with time. Thus, such models are appropriate for studying the spread of disease. Fortunately, several easy-to-use systems dynamics tools, such as STELLA®, Vensim ${ }^{\circledR}$, or Berkeley Madonna ${ }^{\circledR}$, are available to create pictorial representations of models, establish relationships, run simulations, and generate graphs and tables of the results.

In the first week's laboratory in the sequence on modeling, students have an introduction to the fundamental ideas with a predator-prey model using the systems dynamics modeling tool STELLA. The concept of rate of change, or derivative, is crucial to systems dynamics modeling. Even students who have not had calculus or who have not taken mathematics in several years quickly grasp the concept and derivative notation. In the tutorial, they learn that in unconstrained growth, such as for the prey in an environment of unlimited resources and no predator, the rate of change of prey is proportional to the number of prey, or $d($ prey_population $) / d t=$ growth_rate * prey_population, where growth_rate is a constant. Figure 1 shows a model diagram of the prey in such a circumstance. Using systems dynamics software, the user double-clicks each component and enters the initial prey population, constant of proportionality, and differential equation for growth, namely growth_rate * prey_population, in the flow into prey_population. Then, he or she can instruct the tool to generate a table and graph, such as in Table 1 and Figure 2.

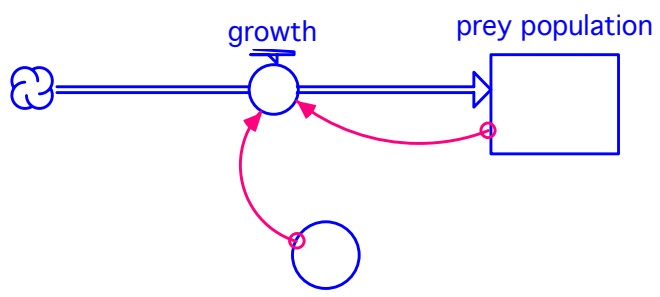

growth rate

Fig. 1. Unconstrained growth diagram [5] 
Table 1. Partial unconstrained growth table

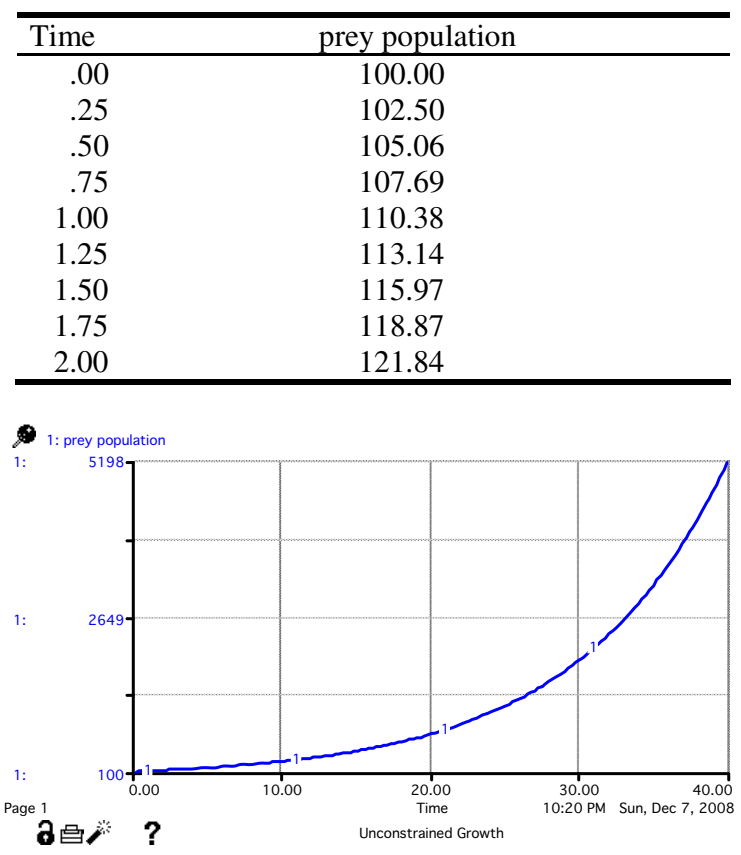

Fig. 2. Unconstrained growth graph

With the introduction of a predator, the model must consider the interaction between predators and prey. The Lotka-Volterra model has the simplifying assumptions that the particular predator only hunts the specific prey and that no other animal eats that prey. Figure 3 shows a systems dynamics diagram for this model.

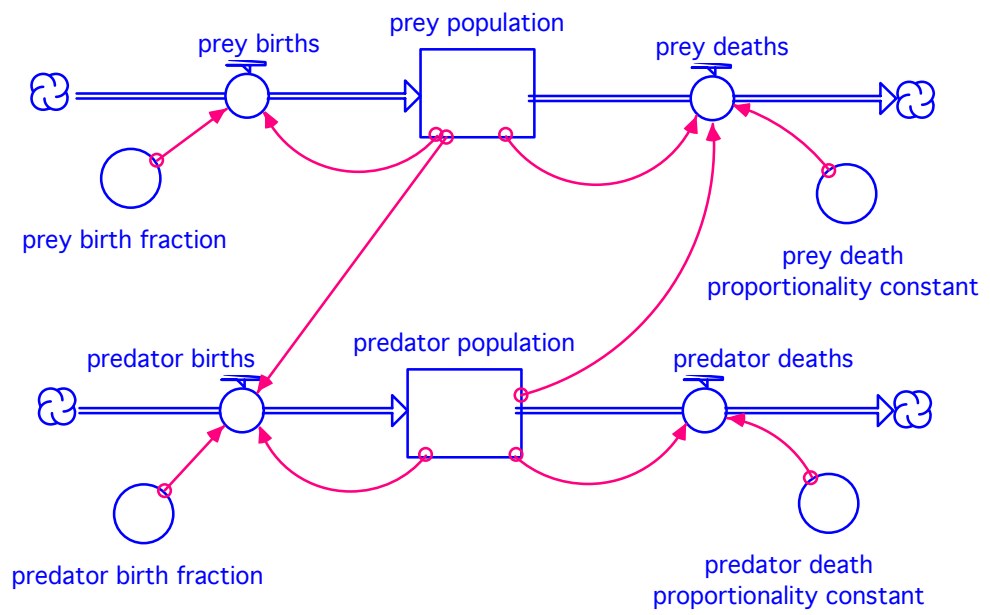

Fig. 3. Predator-prey diagram 
Students quickly grasp that prey births and predator deaths follow the unconstrained growth/decay model. However, this prey's population is reduced by an amount proportional to the product of the number of predators and the prey, prey_death_proportionality_constant * predator_population * prey_population. One interpretation considers the product predator_population * prey_population, which is maximum number of distinct interactions between predators and prey. The decrease in the number of prey is proportional to this product, where the constant of proportionality is related to the hunting ability of the predators and the survival ability of the prey. A second interpretation is that the size of the prey population decreases in proportion to the size of the predator population.

While the prey population decreases with more contacts, the predator population increases. Thus, predator_births is predator_birth_fraction * predator_population * prey_population. Thus, the resulting system of differential equations for the Lotka-Volterra model is as follows, where $p$ is the number of prey, $h$ is the number of predators, and $k_{1}, k_{2}, k_{3}$, and $k_{4}$ are constants:

$$
\begin{aligned}
& \frac{d p}{d t}=k_{1} p-k_{2} h p \\
& \frac{d h}{d t}=k_{3} p h-k_{4} h
\end{aligned}
$$

After considering a predator-prey model, students develop a simple SIR (susceptibles-infecteds-recovereds) model of the spread of disease using a systems dynamics tool, such as STELLA. Figure 4 displays an SIR model diagram. With the analogy of unconstrained growth/decay for prey births and predator deaths, students quickly understand the rate recover to be proportional to infecteds, so that recovery_rate * infecteds is the formula in the recover flow. Moreover, just as interactions hurt prey and help predators, interactions between susceptibles and infecteds result in illness. Thus, the flow from susceptibles to infecteds gets the formula infection_constant * susceptibles * infecteds. Figure 5 presents a typical graph of the number of susceptibles, infecteds, and recovereds for the SIR model.

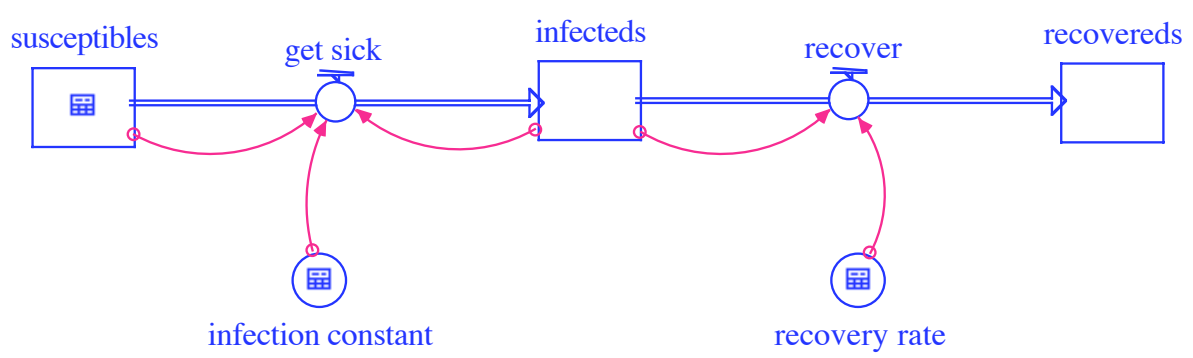

Fig. 4. SIR diagram

During this first lab, a hat is passed around the class for each student to pick a disease. With each disease listed twice, student pairs are formed to investigate their disease before the next laboratory. Between the first and second laboratories, the student pairs ascertain as much as possible about the nature of their assigned diseases, including data, such as rates of change. 


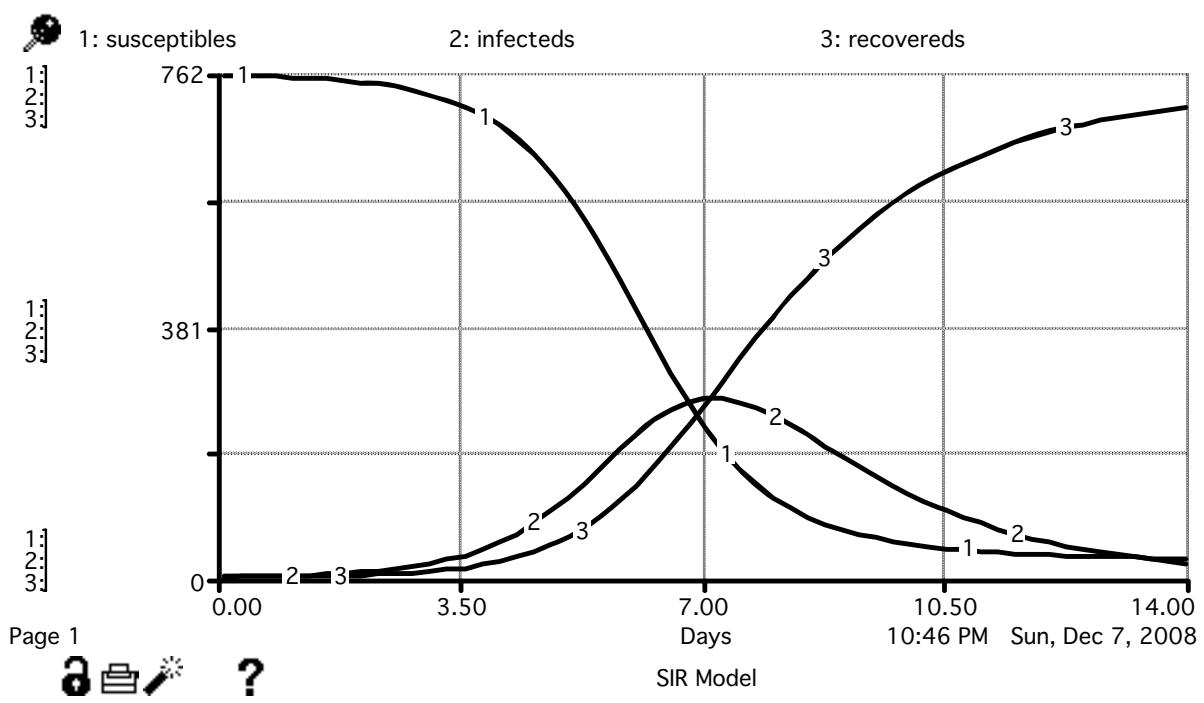

Fig. 5. Graphs for SIR model

\section{Model Development in Laboratories 2 and 3}

In the next two weeks' laboratories with additional time outside of class, each pair develops a model of the spread of their disease using the system dynamics modeling tool. The professor and students obtaining the Emphasis in Computational Science mentor the teams during the laboratory times and outside of class (see Figure 6) [6].

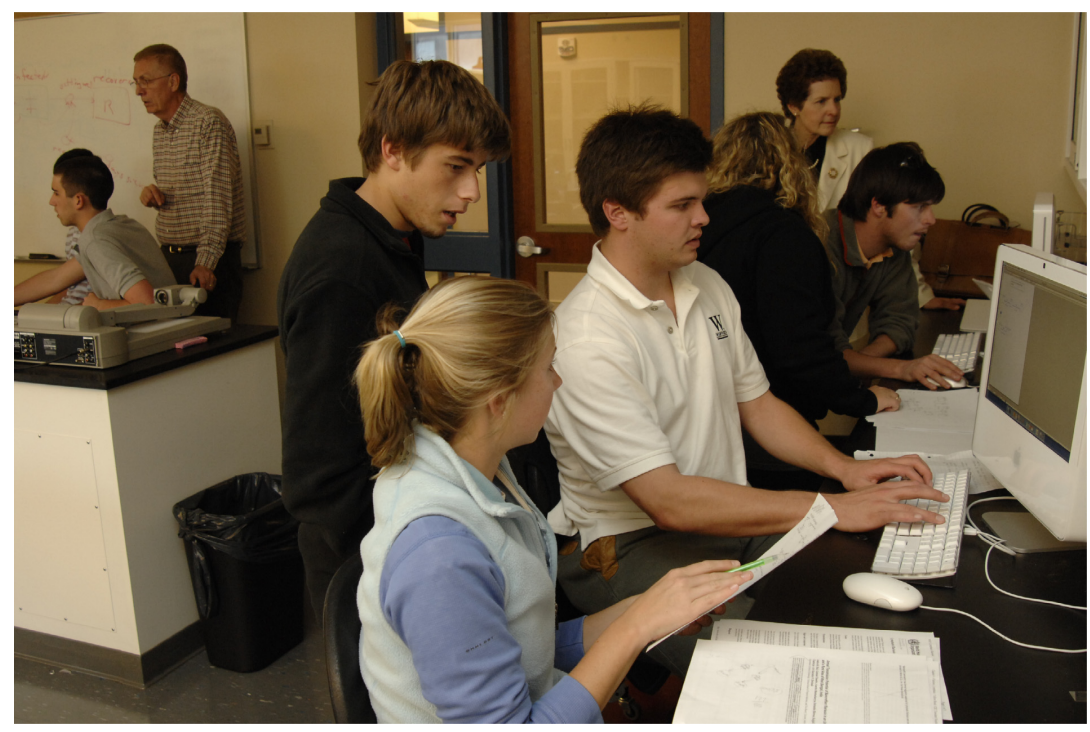

Fig. 6. Student teams 
For example, one pair developed the Chagas disease model whose diagram is in Figure 7. Chagas disease is caused by the protozoa Trypanosomiasis cruzi (T. cruzi), which is usually transmitted by the feces of blood-sucking insect vectors, the "kissing bug". The disease has four main stages in a human: incubation (7-10 days), acute (3-8 weeks), indeterminate, and chronic. The only know drug must be used in the acute phase. This deadly disease infects about 14-million people worldwide, including $20 \%$ of the Bolivian population [7].

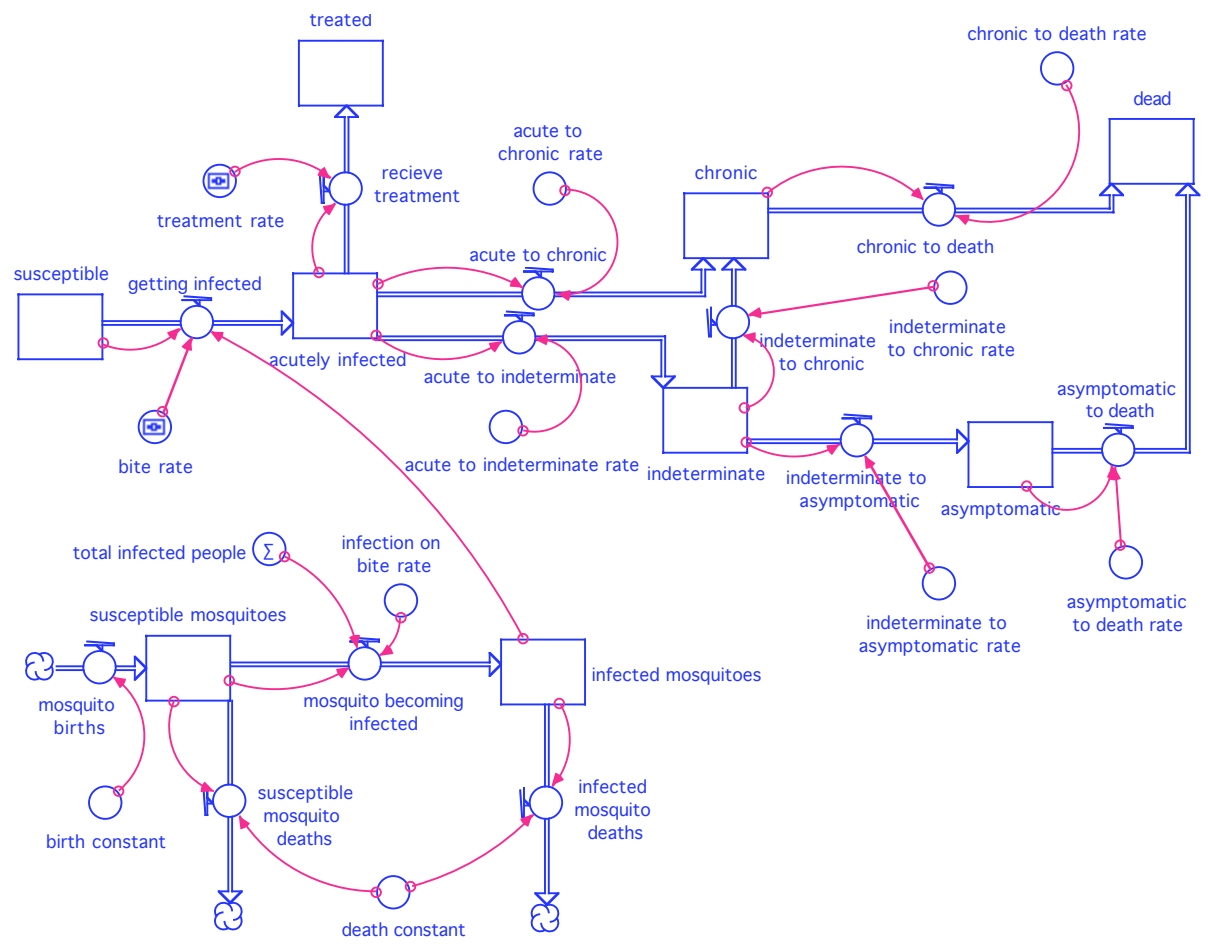

Fig. 7. Diagram model of Chagas disease by student team Lansing Yarborough and Lizzie Dilworth

\section{Presentations in Laboratory 4}

In the final week of the lab sequence, each team makes a 10-to-15 minute presentation on their disease and model to the class. The oral report include the following components:

- General description of the disease, including pertinent details about the agent, how it is spread, factors that affect its spread, signs, symptoms, human impact, general course, treatments, etc.

- General description of your model, including how it functions and what the team considered when designing it

- Demonstration of how the model works 
- Weaknesses and strengths of the model

- Modifications that could be made to improve the model

- Applications of the model

\section{Final Report}

Independently, each student writes a report on his or her team's model and what they learned from the experience. These reports should include the following aspects:

- General description of the disease, which includes the basic biology of the disease (type of organism, symptoms/signs, typical treatments, etc.), a description of what is known about its transmission, and known statistics or rates

- Printout of the model along with a description of the model and how it works, the most important features and the reasons for including them, and simplifying assumptions

- Printout of execution of the model including graphs and tables of several runs of with documentation and justification for the parameters on each run

- Conclusions: What does the model show? What factors does it take into account? What factors seem to be most important to the spread of this disease? What factors does it not take into account and why? How could the model be improved? What applications might be made of the model?

- References for learning about the disease and developing the model along with a briefly critique for utility and quality of each reference

- Self-assessment: How does the individual think he or she performed as a modeler? Did developing the model help the student to understand about the disease and its transmission? How? If not, why was it not helpful?

One or two computational science students help in evaluation of the models and the reports.

\section{Evaluations}

The assessment portions of the final reports reveal a deeper understanding of the spread of their diseases, the modeling process, and the utility of models. The following comments are representative:

- "If I had not already chosen a profession such a long time ago, this [computational biology] would certainly be a possibility. I feel that by designing a model I gained a more comprehensive understanding of Chagas Disease than I would have if I simply had to do a PowerPoint presentation on the subject. The model almost allowed an 'inside-look' at the mechanics of the disease."

- "Developing the model helped me to appreciate the inter-connectedness of all of the factors that influence a disease and helped me to visualize why the disease becomes endemic. I enjoyed the victory of understanding something that initially made little sense to me and frustrated me." 
- "The model definitely helped me to understand more of pneumocystis and its transmission. Only so much of a disease can be understood from a textbook, especially of its complexities. Software as dynamic as Stella makes learning about the way pneumocystis is transmitted so much clearer and more comprehensible. I wasn't only seeing numbers as I would on a page, but literally watching the rates and progress of transmission change before my eyes."

- "Developing this model was very useful in learning about the spread of Lassa Fever. Through research, I was able to learn about this disease while at the same time applying what I learned to the model. Having to make a model based on what you learn makes you very conscious of all the factors that influence the spread of the disease."

- "I did enjoy constructing the model and would definitely enjoy taking on the challenge of developing a model for a much more complicated disease. Developing the model did help me understand the disease and its transmission....I feel that this exercise has been a beneficial experience for me and is an excellent tool for studying disease and epidemiology."

Although evaluations by the students are almost unanimously favorable, improved performance by the students is difficult to quantify. However, on the microbiology final exam, the professor asks questions about disease risks and preventative measures when traveling abroad, and the answers now include in-depth considerations of vector control, water purification, and the other less obvious complicating factors. Before starting the modeling component of the course, students just gave a list of diseases with avoidance behavior suggestions. Moreover, in a subsequent medical case studies class, students present much more comprehensive suggestions to management and prevention issues than previously provided. Thus, the sequence of modeling laboratories appears to accomplish the goals of improving students' understanding of human disease dynamics and the utility of computational models.

\section{Conclusion}

Modeling the spread of disease in a sequence of microbiology laboratories has been beneficial in a number of ways. Students gain an understanding of fundamental concepts, such as rate of change, unconstrained growth, and interactions. Through model development, testing, and refinement, they utilize and improve critical thinking and problem-solving skills. By working with a partner, the students experience teamwork, which is so important to science. Through project oral presentations and written reports, they can improve their communication skills. Moreover, we have found that this interactive learning experience enhances the students' appreciation and understanding of modeling and computational science.

\section{References}

1. Lipsitch, M., et al.: Transmission Dynamics and Control of Sever Acute Respiratory Syndrome. Sciencexpress Report, May 23 (2003), http: / /www.sciencexpress.org/ 23May2003/Page1/10.1126/science. 1086616

2. van Genugten, M.L.L., Heijnen, M.A., Jager, J.C.: Pandemic Influenza and Healthcare Demand in the Netherlands: Scenario Analysis. Emerging Infectious Diseases 9(5), 531-538 (2003) 
3. Models of Infectious Disease Agent Study - National Institute of General Medical Sciences, http://www.nigms.nih.gov/Initiatives/MIDAS /

4. Wofford College, http: / /www. wofford. edu

5. Shiflet, A., Shiflet, G.: Introduction to Computational Science: Modeling and Simulation for the Sciences. Princeton University Press, Princeton (2006)

6. Computational Science, http: / /www. wofford. edu/ecs /

7. Chagas Disease (American Trypanosomiasis): Overview - eMedicine, http://emedicine.medscape.com/article/214581-overview 